\title{
Spatial differences and pattern evolution of Multi-scale Urban Land between China and India
}

\author{
Qinke Sun $^{\text {a, b, c }}$, Liang Zhou ${ }^{\text {a, b, c, }, \text {, Xuewei Dang }}{ }^{\text {a, b, c }}$, Bowei Hu ${ }^{\text {a, b, c }}$, Haowei Mu ${ }^{\text {a, b, c }}$ \\ ${ }^{a}$ Faculty of Geomatics, Lanzhou Jiaotong University, Lanzhou 730070, China; Qinke Sun, qksun2018@163.com, Liang Zhou, \\ zhougeo@126.com, Xuewei Dang,ddangxw@163.com, Bowei Hu,1197180626@qq.com, Haowei Mu, 738242315@qq.com \\ ${ }^{b}$ National-Local Joint Engineering Research Center of Technologies and Applications for National Geographic State Monitoring, \\ Lanzhou 730070, China; Qinke Sun, qksun2018@163.com,Liang Zhou, zhougeo@126.com, Xuewei Dang,ddangxw@163.com, \\ BoweiHu,1197180626@qq.com,HaoweiMu,738242315@qq.com \\ ${ }^{c}$ Gansu Provincial Engineering Laboratory for National Geographic State Monitoring, Lanzhou 730070, China; Qinke Sun, \\ qksun2018@163.com, Liang Zhou, zhougeo@126.com,Xuewei Dang, ddangxw@163.com, Bowei Hu, 1197180626@qq.com, \\ Haowei Mu,738242315@qq.com \\ * Corresponding author
}

Keywords: Spatial pattern, Spatial differences, DMSP-OLS, China, India

\begin{abstract}
:
" The dragon and the elephant " between China and India is an important manifestation of global multipolarization in the 21st century. As an engine of global economic growth, China and India have similar development processes, different development models as well as differences in urban development, which have attracted widespread attention from scholars. Based on the 1992-2012 consecutive annual nighttime lighting data (DMSP-OLS), this paper uses the Gini coefficient, the Getis-Ord Gi* index and the Compounded Night Light Index (CNLI) to conduct a multi-scale comparative analysis of the differences in urban development between China and India from the perspective of geospatial. The results show that: (1) The space of urban land in the two countries expanded rapidly, with an average annual expansion rate of $5.24 \%$ and $3.85 \%$ respectively. China's urban land expansion rate is 1.36 times that of India. Among them, the arid resource towns in northwest China and the resource towns in central India have recently developed rapidly. (2) India's imbalance in development is more prominent than China's. China's regional and provincial imbalances are narrowing, while the regional differences in India are gradually widening. (3) The spatial pattern of land use in both countries shows a certain degree of coastal and inland differences. The main spatial pattern of China's regional development is the difference between East-Central-West, while the spatial pattern of regional development in India is North-South difference. (4) The strength of the expansion of the core cities of the two countries is quite different. From 1997 to 2007, China's core urban expansion intensity remained at a relatively high level while India was at a relatively low level. But from 2007 to 2012, India's core cities expanded at a relatively high level while China was at a relatively low level.
\end{abstract}

